\title{
Different ways of modeling spatial-frequency uncertainty in visual signal detection
}

\author{
Ronald Hübner \\ Institut für Psychologie, Technische Universität Braunschweig, Spielmannstrasse 19, D-38106 Braunschweig, Germany
}

\begin{abstract}
Inferior human signal-detection behavior compared with that of ideal observers has been explained by intrinsic uncertainty of the human observer with respect to certain signal parameters. One way to model this uncertainty is to assume that the observer simultaneously monitors multiple channels, corresponding to possible parameters. However, it is also conceivable to assume that an observer, uncertain about which channel to monitor, chooses a suboptimally tuned single filter. Finally, uncertainty may also cause the filter underlying a single channel to broaden. In this paper these different models are investigated with respect to spatial-frequency uncertainty for matched filters detecting Gabor signals. All three mechanisms predict a decrease in detection performance. However, it is shown that the resulting psychometric functions are different. While the slopes increase with uncertainty for the multiple-channel models, they decrease for a randomly chosen single channel. Broadening a single filter leads to parallel psychometric functions.
\end{abstract}

\section{Introduction}

It is well known that signal-detection performance suffers from randomly varying signal parameters, compared with a situation with fixed parameters. Such an uncertainty effect has been demonstrated in visual signal detection for various parameters such as phase (Burgess and Ghandeharian 1984a), location (Swensson and Judy 1981; Davis et al. 1983; Burgess and Ghandeharian 1984b), and spatial frequency (Davis et al. 1983). This paper is concerned mainly with spatial-frequency uncertainty.

Two classes of models have been proposed to explain the observed uncertainty effects: multiple-band and singleband models. Multiple-band models assume that observers simultaneously monitor several channels or bands when they are uncertain about a signal parameter. For instance, if the task is to detect a sinusoidal grating with unknown spatial frequency, then it is assumed that an observer monitors the output of multiple frequencyspecific filters or channels. Since this strategy increases the number of false alarms, the decrease in detection performance can be explained.
Single-band models, on the other hand, assume that the monitoring capacity is limited to one channel at a time. Here, when there is uncertainty about the spatial frequency of the signal, the observer is assumed to switch between different channels from trial to trial. Since this strategy leads to inappropriately tuned channels in some trials, a decrease in detection performance is predicted. If it is further assumed that after a correct response the observer does not switch to another channel, then sequential dependencies are also to be expected [see Swets (1984) or Graham (1989) for more details].

Unfortunately, the empirical results are inconclusive. While uncertainty effects were usually smaller than predicted by the single-band models, sequential dependencies were found (Davis and Graham 1981). Multiple-band models, on the other hand, predict the size of the effect more precisely. However, they additionally predict a steepening of psychometric functions collected under uncertainty which could not be confirmed for spatial-frequency uncertainty (Graham 1989, p 303).

Single-band models play a further role in connection with the so-called probe-signal technique. Experiments employing this method also induce spatial-frequency uncertainty, but with unbalanced presentation frequencies. A primary signal with a certain spatial frequency is presented in the majority of the trials. In the remaining trials, probe signals with different spatial frequencies are presented. Signal levels are chosen to produce equal detectability in blocked presentations. Results obtained with the probe-signal method show that the detectability of the probes decreases with distance from the spatial frequency of the primary signal (Davis and Graham 1981). This is thought to reflect the tuning curve of the utilized internal filter centered at the spatial frequency of the primary signal.

This interpretation rests on the assumption that frequent presentation of the primary signal is sufficient to tune the utilized channel to the spatial frequency of that signal. However, as has been demonstrated by Davis and Graham (1981), the "tuning" depends on the proportion of the primary signal. As the proportion increased from 80 to $95 \%$ tuning became sharper and more centered at the primary spatial frequency. How can this result be explained? Davis and Graham (1981) speculated that in 
the $80 \%$ condition additional spatial-frequency channels were monitored and that only in the $90 \%$ condition was a stationary single band employed. However, it is also conceivable that in the $80 \%$ condition the filter was not exactly tuned to the spatial frequency of the primary signal at every trial and that the increased proportion of the probes could have led to filter jitter. That off-frequency looking can occur in some situations has been shown, for example, by Perkins and Landy (1991).

A third alternative is to assume that the employed band is variable in width and that the $80 \%$ condition induces the perceptual filter to broaden (Johnson and Hafter 1980).

Thus far, so-called extrinsic uncertainty was concerned, which is deliberately introduced by presenting signals with random parameters to the observer. However, it is also believed that there is some intrinsic uncertainty. This means that even if no extrinsic uncertainty is present, human observers will never know the signal exactly. In connection with multiple-band models this assumption implies that even with constant signal parameters, observers always monitor more than one channel simultaneously (Graham 1989).

Intrinsic uncertainty has also been used to explain the performance difference between human and ideal observers. For instance, Pelli (1985) was quite successful in explaining several phenomena of visual signal detection by assuming a multiple-band ideal observer degraded by intrinsic uncertainty. However, there are conceivable alternative ways to model intrinsic uncertainty or to explain the reduced detection performance of human observers, compared with ideal observers, particularly, if one assumes a single-band model. For such models it would also be reasonable to establish intrinsic uncertainty by assuming filter jitter or, alternatively, more broadly tuned filters.

However, the effects of these alternative mechanisms do not seem to be clear. Swensson and Judy (1981) modeled inappropriate tuning by adding internal noise to an ideal observer. Since ideal observers face only external noise, adding internal noise would seem to be a suitable method of degrading their performance (Burgess and Colborne 1988). However, it will be demonstrated that adding internal noise to model mistuning is not adequate.

As has been shown, there are several occasions where filter tuning seems to be important. However, we have hardly any knowledge about the effects of filter mistuning, except that it degrades detection performance, which is insufficient in most cases to distinguish it from other mechanisms. Since it is quite characteristic for the multiple-band models that the slopes of the psychometric functions increase with the number of bands, it would be desirable to consider psychometric functions of a randomly tuned single band for comparison. In this paper such psychometric functions are derived for ideal observers detecting sinusoidal and Gabor signals, degraded by filter jitter.

Additionally, psychometric functions for ideal observers with increased bandwidth are provided.

\section{Matched filtering in a 2IFC situation}

Although visual signal processing is mainly concerned with two-dimensional stimuli, we will consider only onedimensional signals. This makes the analysis much easier while still obtaining relevant results.

Assume that the task is to detect a signal $s(x)$ in given input data $\mathbf{i}(x)$ which can be either noise $\mathbf{n}(x)$ or signal plus noise, i.e.,

$$
\begin{aligned}
& \mathbf{i}_{s}(x)=s(x)+\mathbf{n}(x) \\
& \mathbf{i}_{n}(x)=\mathbf{n}(x)
\end{aligned}
$$

Assume further that the noise is a normal process, i.e., $\mathbf{n}(x)$ is distributed as $N\left(0, N_{0}\right)$, and that the signal is known exactly. The effectiveness of a detector depends on the signal-to-noise ratio, i.e., the ratio between the value $s\left(x_{0}\right)$ of $s(x)$ at a certain point $x_{0}$ and the standard deviation of the noise. This ratio can be increased if the data are processed by a linear filter $H$ whose output $y(x)$ with input $i(x)$ is given as

$$
\begin{aligned}
y(x) & =\frac{1}{2 \pi} \int_{-\infty}^{\infty} I(\omega) H(\omega) \mathrm{e}^{j \omega x} \mathrm{~d} \omega \\
& =\int_{-\infty}^{\infty} i(u) h(x-u) \mathrm{d} u
\end{aligned}
$$

where $h(x)$ is the impulse-response function of the filter $H(\omega)$ and $I(\omega)$ is the Fourier transform of $i(x)$.

It can be shown that a filter, maximizing the signalto-noise ratio, is given by (Papoulis 1984)

$H(\omega)=k S^{*}(\omega) \mathrm{e}^{-j \omega x_{0}}$

where $k$ is an arbitrary constant and $S^{*}$ denotes the complex conjugate of $S$. Such a system is called a matched filter for the signal $s(x)$, because its impulse-response function is $h(x)=k s^{*}\left(x_{0}-x\right)$.

Because the signal-plus-noise input is a sum and the filter is linear, we can also write its output as a sum:

$$
\begin{aligned}
\mathbf{y}_{s}(x)= & \int_{-\infty}^{\infty} s(u) h(x-u) \mathrm{d} u+\int_{-\infty}^{\infty} \mathbf{n}(u) h(x-u) \mathrm{d} u \\
= & k \int_{-\infty}^{\infty} s(u) s^{*}\left(x_{0}-x+u\right) \mathrm{d} u \\
& +k \int_{-\infty}^{\infty} \mathbf{n}(u) s^{*}\left(x_{0}-x+u\right) \mathrm{d} u
\end{aligned}
$$

We assume that a detector in a 2IFC (two-interval forced-choice) situation draws two samples, $\mathbf{U}_{s}$ and $\mathbf{U}_{n}$, corresponding to signal plus noise and noise alone and chooses the interval containing the larger sample. This behavior corresponds to a maximum-likelihood strategy. The detector is correct if the larger sample is from the signal-plus-noise distribution. Thus, the proportion of correct detection is given by

$P\{C\}=P\left\{\mathbf{U}_{s}>\mathbf{U}_{n}\right\}=\int_{-\infty}^{\infty} F_{n}(u) f_{s}(u) \mathrm{d} u$

where $F_{n}(u)=\int_{-\infty}^{u} f_{n}(y) \mathrm{d} y$ is the distribution function of $\mathbf{U}_{n}$ and $f_{s}(u)$ the probability function of $\mathbf{U}_{s}$. 
Fortunately, since $\mathbf{i}(x)$ is a normal process, so is $\mathbf{y}(x)$ (Papoulis 1984). We will evaluate the mean and variance of the noise-alone and signal-plus-noise distributions.

Since the maximum output of the filter is independent of the position of the signal, we consider its performance at $x_{0}=0$. For the noise-alone case one obtains as mean

$\mathscr{E}\left\{\mathbf{y}_{n}\left(x_{0}\right)\right\}=\int_{-\infty}^{\infty} \mathscr{E}\{\mathbf{n}(u)\} h\left(x_{0}-u\right) \mathrm{d} u=0$

and as variance (Papoulis 1984)

$$
\begin{aligned}
\operatorname{Var}\left\{\mathbf{y}_{n}\left(x_{0}\right)\right\} & =\frac{1}{2 \pi} \int_{-\infty}^{\infty} I_{n}(\omega)|H(\omega)|^{2} \mathrm{~d} \omega \\
& =\frac{k^{2} N_{0}}{2 \pi} \int_{-\infty}^{\infty}\left|S^{*}(\omega)\right|^{2} \mathrm{~d} \omega \\
& =k^{2} N_{0} E_{s}
\end{aligned}
$$

The output of a matched filter for signal-plus-noise at $x_{0}$ is given by

$$
\begin{aligned}
\mathbf{y}_{s}\left(x_{0}\right) & =k \int_{-\infty}^{\infty} s(u) s^{*}(u) \mathrm{d} u+k \int_{-\infty}^{\infty} \mathbf{n}(u) s^{*}(u) \mathrm{d} u \\
& =k E_{s}+k \int_{-\infty}^{\infty} \mathbf{n}(u) s^{*}(u) \mathrm{d} u
\end{aligned}
$$

where $E_{s}$ denotes the energy of the signal. Therefore, one obtains as mean for the signal-plus-noise distribution

$\mathscr{E}\left\{\mathbf{y}_{s}\left(x_{0}\right)\right\}=k E_{s}$

and as variance

$\operatorname{Var}\left\{\mathbf{y}_{s}\left(t_{0}\right)\right\}=k^{2} N_{0} E_{s}$

An equivalent interpretation of the detector's performance is to say that its choice is correct if $\mathbf{U}_{s}-\mathbf{U}_{n}>0$. Since the two variables are normal, their difference is also normally distributed. Thus, we get

$P\{C\}=\Phi(z)=\frac{1}{\sqrt{2 \pi}} \int_{-\infty}^{z} \mathrm{e}^{-\frac{u^{2}}{2}} \mathrm{~d} u$

with

$$
z=\sqrt{\frac{E_{s}}{2 N_{0}}}=\frac{d^{\prime}}{\sqrt{2}}
$$

The index of sensitivity, $d^{\prime}$, represents the difference in the means of the noise and signal-plus-noise distributions divided by their common standard deviation:

$d^{\prime}=\frac{E_{s}}{\sqrt{N_{0} E_{s}}}=\sqrt{\frac{E_{s}}{N_{0}}}$

This differs from the result $\sqrt{2 E_{s} / N_{0}}$, usually derived for auditory signal detection, because we used two-sided spectra.

\section{Effects of filter displacements}

Thus far we have considered the usual detection behavior of a matched filter. In this section we will investigate the

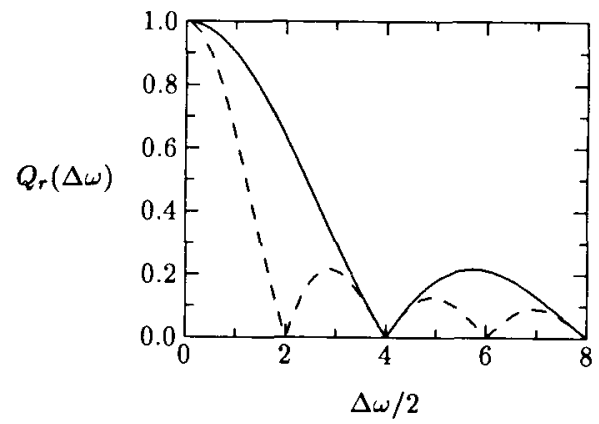

Fig. 1. Function $Q,(\Delta \omega)$. The solid curve corresponds to $L=\pi / 4$ and the dashed curve to $L=\pi / 2$

effects of its mistuning. Therefore, we consider its output in dependence on the amount of displacement:

$$
\begin{aligned}
\mathbf{y}_{s}(x, \Delta \omega)= & \frac{1}{2 \pi} \int_{-\infty}^{\infty} I(\omega) H(\omega+\Delta \omega) \mathrm{e}^{j \omega x} \mathrm{~d} \omega \\
= & \frac{1}{2 \pi} \int_{-\infty}^{\infty} S(\omega) H(\omega+\Delta \omega) \mathrm{e}^{j \omega x} \mathrm{~d} \omega \\
& +\frac{N_{0}}{2 \pi} \int_{-\infty}^{\infty} H(\omega+\Delta \omega) \mathrm{e}^{j \omega x} \mathrm{~d} \omega
\end{aligned}
$$

By noticing that the noise part is not affected by a displacement and using the fact

$\frac{1}{2 \pi} \int_{-\infty}^{\infty} H(\omega+\Delta \omega) \mathrm{e}^{j \omega x} \mathrm{~d} \omega=h(x) \mathrm{e}^{-j \Delta \omega x}$

we get for the signal part at $x_{0}$

$$
\begin{aligned}
y^{s}\left(x_{0}, \Delta \omega\right) & =\int_{-\infty}^{\infty} s(u) h\left(x_{0}-u\right) \mathrm{e}^{-j \Delta \omega\left(x_{0}-u\right)} \mathrm{d} u \\
& =\int_{-\infty}^{\infty}|s(u)|^{2} \mathrm{e}^{-j \Delta \omega(u)} \mathrm{d} u
\end{aligned}
$$

To describe the loss in effectivity of a suboptimal filter we introduce a function $Q$ which is one when optimality is given. In the case considered here, we have optimality if $\Delta \omega=0$. Thus,

$Q(\Delta \omega)=\frac{\int_{-\infty}^{\infty}|s(u)|^{2} \mathrm{e}^{-j \Delta \omega u} \mathrm{~d} u}{\int_{-\infty}^{\infty}|s(u)|^{2} \mathrm{~d} u}$

\subsection{Rectangular signals}

Now let us specify a certain signal. Vander Lugt (1967) has shown that the signal with

$|s(x)|^{2}= \begin{cases}1, & \text { for } 0 \leqslant x \leqslant L \\ 0, & \text { elsewhere }\end{cases}$

has the most rapid loss of performance for small values of $\Delta \omega$. Applying this signal to (3) gives

$Q_{r}(\Delta \omega)=\frac{\sin (\Delta \omega L / 2)}{\Delta \omega L / 2}$

Figure 1 shows the function $Q_{r}(\Delta \omega)$ for different values of $L$. As one can see, the quotient drops faster for 


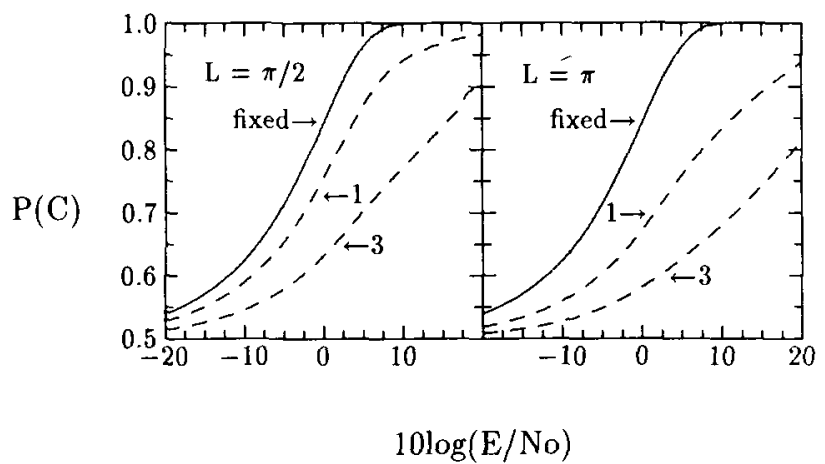

Fig. 2. Theoretical psychometric functions of a matched-filter detector. The solid curves reflect a fixed filter. The dashed curves reflect normally varying filters with standard deviations $\sigma=1$ and $\sigma=3$, respectively

long signals. This is because the spectrum becomes narrower for longer signals.

Next, we will apply this function to the ideal observer. We assume that the filter position is fixed within a trial but varies randomly from trial to trial (filter jitter). Specifically, we assume that the displacement is a random variable $\mathbf{\Omega}$, which is normally distributed with $N(0, \sigma)$ :

$f(\Delta \omega)=\frac{1}{\sqrt{2 \pi} \sigma} \mathrm{e}^{-\frac{(\Delta \omega)^{2}}{2 \sigma^{2}}}$

The proportion of correct detection is now conditioned on the displacement:

$P^{\prime}\{C\}=\int_{-\infty}^{\infty} P\{C \mid \boldsymbol{\Omega}=\Delta \omega\} f(\Delta \omega) \mathrm{d} \Delta \omega$

where

$P\{C \mid \boldsymbol{\Omega}=\Delta \omega\}=\Phi\left[z_{r}(\Delta \omega)\right]$

with

$z_{r}(\Delta \omega)=Q_{r}(\Delta \omega) \sqrt{\frac{E_{s}}{2 N_{0}}}$

By applying (4) we can compute psychometric functions for several values of $L$ and $\sigma$. Figure 2 depicts some examples.

As one can see, introducing filter jitter shifts the psychometric functions to the right, i.e., performance is decreased. Additionally, the slopes of the psychometric functions decrease when the variance of the filter displacement increases.

\subsection{Gabor signals}

In this section we consider Gabor signals. The corresponding matched filters are Gabor filters (Daugmann 1985) which are quite popular as models for visual receptive fields. For convenience, we consider one-dimensional Gabor signals which have the form

$g\left(x-x_{0}\right)=\mathrm{e}^{-\frac{\left(x-x_{0}\right)^{2}}{2 \eta^{2}}} \mathrm{e}^{j \omega_{0}\left(x-x_{0}\right)}$

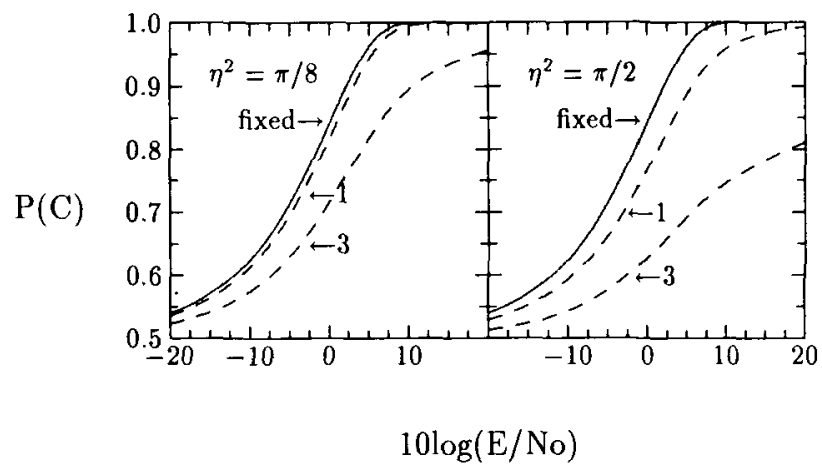

Fig. 3. Theoretical psychometric functions of matched Gabor filters. The solid curves reflect a fixed filter. The dashed curves reflect normally varying filters with standard deviations $\sigma=1$ and $\sigma=3$, respectively

The Fourier transform is given by

$G\left(\omega-\omega_{0}\right)=\sqrt{2 \pi} \eta \mathrm{e}^{-\frac{\left(\omega-\omega_{0}\right)^{2}}{2 \tau^{2}}} \mathrm{e}^{-j x_{0}\left(\omega-\omega_{0}\right)}$

Here, $\eta$ is the spatial standard deviation and $\tau$ the spatial-frequency standard deviation with $\tau=1 /(2 \pi \eta)$. For convenience, we again consider the case with $x_{0}=0$. Applying this Gabor signal to (3) gives

$$
\begin{aligned}
Q_{g}(\Delta \omega) & =\frac{\int_{-\infty}^{\infty} \mathrm{e}^{-\frac{x^{2}}{\eta^{2}}} \mathrm{e}^{-j \Delta \omega x} \mathrm{~d} x}{\int_{-\infty}^{\infty} \mathrm{e}^{-\frac{x^{2}}{\eta^{2}}} \mathrm{~d} x} \\
& =\frac{\mathrm{e}^{-\frac{\Delta \omega^{2} \eta^{2}}{4} \int_{-\infty}^{\infty} \mathrm{e}^{-\frac{\left(x-j \Delta \omega \eta^{2}\right)^{2}}{\eta^{2}}} \mathrm{~d} x}}{\int_{-\infty}^{\infty} \mathrm{e}^{-\frac{x^{2}}{\eta^{2}}} \mathrm{~d} x} \\
& =\mathrm{e}^{-\frac{(\Delta \omega \eta)^{2}}{4}}
\end{aligned}
$$

In this case the function $Q_{g}$ is exponential and therefore monotonically decreasing.

By applying (4) with

$z_{g}(\Delta \omega)=Q_{g}(\Delta \omega) \sqrt{\frac{E_{s}}{2 N_{0}}}$

we can compute psychometric functions for Gabor signals with several values of $\eta$ and $\sigma$. Figure 3 depicts some examples using Gabor signals covering equivalent areas under their impulse-response functions as the rectangular signals in Fig. 2.

As one can also see in this case, the psychometric functions shift to the right, and the slopes decrease if the variance of the filter position increases. However, the loss is not as severe as with the rectangular filters.

\section{Increasing filter width}

In this section the effect of increasing the width of a matched filter on signal detection performance is considered. We again use Gabor signals. For convenience we 
let $\omega_{0}=0, x_{0}=0$, and $\tau=1$ without restricting generality. The model consists of increasing the filter width by increasing the spatial-frequency standard deviation. This is equivalent to scaling the space domain, i.e., multiplying the coordinate $x$ by a factor $a$, with $a \geqslant 1$, to get $g(a x)$. This narrows the signal and, consequently, increases the width of its spectrum. For the signal part we can describe the effectivity by

$$
\begin{aligned}
Q_{w}^{s}(a) & =\frac{\frac{1}{2 \pi|a|} \int_{-\infty}^{\infty} G(\omega) G(\omega / a) \mathrm{d} \omega}{\frac{1}{2 \pi} \int_{-\infty}^{\infty}|G(\omega)|^{2} \mathrm{~d} \omega} \\
& =\frac{\frac{1}{|a|} \int_{-\infty}^{\infty} \mathrm{e}^{-\frac{\omega^{2}}{2 \frac{a^{2}}{a^{2}+1}}} \mathrm{~d} \omega}{\int_{-\infty}^{\infty} \mathrm{e}^{-\omega^{2}} \mathrm{~d} \omega} \\
& =\sqrt{\frac{2}{a^{2}+1}}
\end{aligned}
$$

In contrast to shifting a filter in spatial-frequency domain, in this case effectivity of the noise part is also affected. Widening the filter increases the variance of the output. Thus, we get for the noise part

$$
\begin{aligned}
Q_{w}^{n}(a) & =\frac{\frac{N_{0}}{2 \pi|a|^{2}} \int_{-\infty}^{\infty}|G(\omega / a)|^{2} \mathrm{~d} \omega}{\frac{N_{0}}{2 \pi} \int_{-\infty}^{\infty}|G(\omega)|^{2} \mathrm{~d} \omega} \\
& =\frac{\frac{1}{|a|^{2}} \int_{-\infty}^{\infty} \mathrm{e}^{-\frac{\omega^{2}}{a^{2}}} \mathrm{~d} \omega}{\int_{-\infty}^{\infty} \mathrm{e}^{-\omega^{2}} \mathrm{~d} \omega} \\
& =\frac{1}{a}
\end{aligned}
$$

Finally, combining both quotients with respect to our decision variable $z$ gives

$Q_{w}(a)=\sqrt{\frac{2 a}{a^{2}+1}}$

To compute psychometric functions (2) can be used. However, instead of $z$ we use $Q_{w}(a) z$. In $\log$ coordinates this is simply a shift by a constant. Therefore, the psychometric functions are parallel. In Fig. 4 we see some examples for different values of $a$.

\section{Increasing the number of monitored filters}

As mentioned before, uncertainty in visual signal detection has already been modeled on the assumption that more than one filter is applied. While displacing as well as widening a filter affects sensitivity, utilizing multiple filters is related to the decision part of the observer. Several methods of combining the outputs of multiple

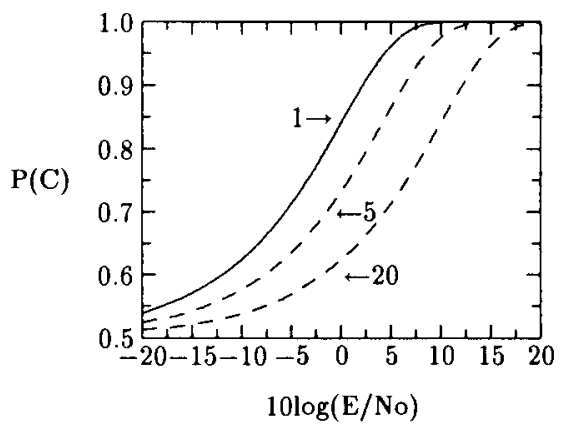

Fig. 4. Psychometric functions resulting from increasing the filter width of a Gabor filter. The solid curve corresponds to $a=1$ and the dashed curves to $a=5$ and $a=20$, respectively

independent filters have been proposed. The optimal way would be to combine their likelihoods. However, this is formally difficult to handle. It is easier to choose the maximum filter output, a strategy first suggested by Creelman (1960) and also applied by Pelli (1985). For a 2IFC task this means that the interval is chosen which contains the maximum value of the filter outputs. Both methods lead to quite similar results (Nolte and Jaarsma 1966; Green and Birdsall 1978).

For the maximum strategy and a 2IFC task we have (Hübner 1993)

$P\{C\}=\frac{M-1}{2 M-1}+\frac{M}{2 M-1} \int_{-\infty}^{\infty} f_{s}(u) F_{n}(u)^{2 M-1} \mathrm{~d} u$

where $M$ indicates the number of filters.

Equation (5) can be used to compute the effects of increasing the number of filters. Examples for a matched filter are depicted in Fig. 5. As one can see, increasing $M$ has the effect of increasing the slope of the corresponding psychometric functions.

\section{Discussion}

It has been shown that a decrease in detection performance caused by spatial-frequency uncertainty can be explained by at least three different mechanisms. Not

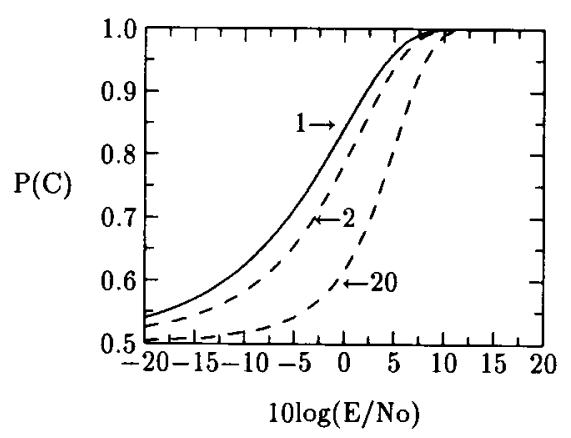

Fig. 5. Theoretical psychometric functions of a detector with multiple matched filters. The digits indicate the number of filters 
only multiple-filter models may account for a loss in efficiency. A single filter whose position varies in spatialfrequency domain from trial to trial may also be appropriate. Finally, a strategy for signal detection under uncertainty might be to employ a filter with a relatively broad bandwidth.

All three mechanisms predict a decrease in detection performance. However, as has been shown here, there are distinguishing differences between them. While for a randomly varied filter position the slopes of the psychometric functions decrease with increasing uncertainty, they increase for multiple filters. Widening a filter produces parallel psychometric functions. Thus, it should be possible to distinguish experimentally between the proposed sources of the observed uncertainty effects. For instance, by collecting psychometric functions of the primary signal in probesignal experiments it should be possible to find out what mechanism produced the different results for different proportions of the primary signal in the experiment of Davis and Graham (1981).

As mentioned in the introduction, ideal observers only have to cope with external noise. Thus, to compare ideal and human observers, external noise can also be presented to human observers as, for example, in the experiments of Burgess and Ghandeharian (1984a, b) and Swenson and Judy (1981). Then either it can be assumed that the internal noise is negligible or it can be added to the model. If internal noise $N_{i}$ is added simply as

$d^{\prime}=\sqrt{\frac{E_{s}}{N_{0}+N_{i}}}$

it is easy to show that this also leads to parallel-shifted psychometric functions. Therefore, mistuning is not adequately modeled by adding internal noise, as done by Swenson and Judy (1981).

The results derived in this paper rely on signal-detection theory, ideal observers, and matched filters. What can be said about alternative models and assumptions? A rather popular model of visual signal detection is high-threshold theory combined with probability summation. However, not only has this model been shown to be invalid (Nachmias 1981; Mortensen 1988), it also predicts no uncertainty effects (Graham 1989). Therefore, it seems to be almost unavoidable but to use signaldetection theory (Green and Swets 1966) for modeling uncertainty effects.

For signals more complex than simple gratings, matched-filter theory (Hauske et al. 1976; Burgess and Ghandeharian 1984a, b; Mortensen 1988) competes with the assumption of multiple tuned channels (Campbell and Robson 1968; Graham 1989). For the latter it is often assumed that the channels correspond to oriented receptive fields which can be modeled by matched filters for Gabor signals. Therefore, as far as the detection of such signals is concerned, the results derived here are valid for both models.
Finally, it should be mentioned that if energy detectors (Green and Swets 1966) are used, the results for a randomly tuned single filter and for multiple filters are similar to those derived here (Hübner 1993). However, the results of widening a filter differ. For the energy detector the psychometric functions are not parallel in this case. Rather, increasing filter width increases the slope. This is due to the fact that for an energy detector output variance also depends on signal energy.

Acknowledgements. This research was supported by a grant ( $\mathrm{Hu}$ 432/2-1) from the Deutsche Forschungsgemeinschaft (DFG).

\section{References}

Burgess A, Colborne B (1988) Visual signal detection. IV. Observer inconsistency. J Opt Soc Am [A] 5:617-627

Burgess A, Ghandeharian H (1984a) Visual signal detection. I. Ability to use phase information. J Opt Soc Am [A] 1:900-905

Burgess A, Ghandeharian H (1984b) Visual signal detection. II. Signallocation identification. J Opt Soc Am [A] 1:906-910

Campbell FW, Robson JG (1968) Application of Fourier analysis to the visibility of gratings. J Physiol (Lond) 197:551-566

Creelman CD (1960) Detection of signals of uncertain frequency. J Acoust Soc Am 32:805-810

Daugman J (1985) Uncertainty relation for resolution in space, spatial frequency, and orientation optimized by two-dimensional visual cortical filters. J Opt Soc Am [A] 2:1160-1169

Davis T, Graham N (1981) Spatial frequency uncertainty effects in the detection of sinusoidal gratings. Vision Res 21:705-712

Davis T, Kramer P, Graham N (1983) Uncertainty about spatial frequency, spatial position, or contrast of visual patterns. Percept Psychophys 33:20-28

Graham N (1989) Visual pattern analysers. Oxford University Press, New York

Green DM, Birdsall TG (1978) Detection and recognition. Psychol Rev 85:192-206

Green DM, Swets JA (1966) Signal detection theory and psychophysics. Wiley, New York

Hauske G, Wolf W, Lupp U (1976) Matched filters in human vision. Biol Cybern 22:181-188

Hübner R (1993) On possible models of attention in signal detection. J Math Psychol 37:266-281

Johnson DM, Hafter ER (1980) Uncertain-frequency detection: cueing and condition of observation. Percept Psychophys 28:143-149

Mortensen U (1988) Visual contrast detection by a single channel versus probability summation among channels. Biol Cybern 59:137-147

Nachmias J (1981) On the psychometric function for contrast detection. Vision Res 21:215-224

Nolte LW, Jaarsma D (1966) More on the detection of one of $m$ orthogonal signals. J Acoust Soc Am 41:497-505

Papoulis A (1984) Probability, random variables, and stochastic processes (3rd edn). McGraw-Hill, New York

Pelli DG (1985) Uncertainty explains many aspects of visual contrast detection and discrimination. J Opt Soc Am [A] 2:1508-1532

Perkins ME, Landy MS (1991) Nonadditivity of masking by narrowband noises. Vision Res 31:1053-1065

Swensson RG, Judy PF (1981) Detection of noisy visual targets: models for the effects of spatial uncertainty and signal-to-noise ratio. Percept Psychophys 29:521-534

Swets JA (1984) Mathematical models of attention. In: Parasuraman R, Davis D (eds) Varieties of attention. Academic Press, New York, pp 183-242

Vander Lugt A (1967) The effects of small displacements of spatial filters. Appl Opt 6:1221-1226 